\title{
Spectroscopically Resolved Binding Sites for the Adsorption of Sarin Gas in a Metal- Organic Framework: Insights Beyond Lewis Acidity
}

Jacob A. Harvey, ${ }^{\dagger}$ Monica L. McEntee, ${ }^{\ddagger}$ Sergio J. Garibay, ${ }^{\ddagger}$ Erin M. Durke,${ }^{\ddagger}$ Jared B. Decoste, ${ }^{\ddagger}$ Jeffery A. Greathouse, ${ }^{\dagger}$ Dorina F. Sava Gallis ${ }^{{ }^{*}}$

Geochemistry Department, Sandia National Laboratories, Albuquerque, New Mexico 87125, USA

* Combat Capabilities Development Command Chemical Biological Center, U.S. Army

Research, Development and Engineering Command, 8198 Blackhawk Road, Aberdeen Proving Ground, MD 21010, USA

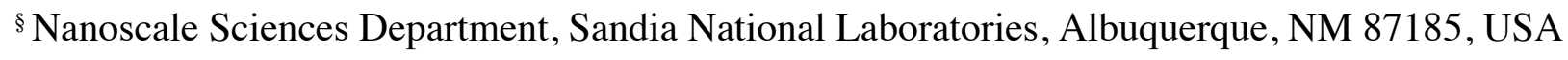

*Corresponding Author: dfsava@sandia.gov 


\section{Input Files for Calculations}

Input files for all calculations discussed in manuscript are given here. VASP POSCAR files used to start optimizations are given along with relevant INCAR files for the optimization and frequency calculations. The final structure from the optimization are used to start the frequency calculations and are not given. Calculated spectra for deuterated GB were performed using the optimized protonated structure and therefore not given but can be generated by changing the mass of the $D$ atoms in the relevant POTCAR file.

GB at defect (C orientation):

POSCAR file from uio-66-prim-defect-gb-1-c.res: C: 44 F: 1 H: 36 O: 32 P: 1 ZR: 6 14.6640000000

$\begin{array}{lll}0.8119283518 & 0.2940430090 & 0.5049819426 \\ 0.0000000000 & 0.8679239315 & 0.5037576415 \\ 0.0000000000 & 0.0000000000 & 1.0000000000\end{array}$

441363216

Direct

$\begin{array}{lll}0.92177 & 0.44699 & 0.08830 \\ 0.08871 & 0.44750 & 0.92110 \\ 1.57875 & -0.11460 & 0.39728 \\ 1.65481 & -0.09389 & 0.42315 \\ 0.92119 & 0.54156 & 0.08824 \\ 1.70886 & -0.20216 & 0.50523 \\ 1.65047 & 0.14631 & 0.50062 \\ 0.53562 & 0.43993 & 0.94044 \\ 0.00215 & 0.69240 & 0.00286 \\ 0.00409 & 0.58960 & 0.00434 \\ 0.00411 & 0.30036 & 0.00305 \\ 0.00525 & 0.39991 & 0.00447 \\ 0.68830 & 0.29318 & 0.01724 \\ 0.58459 & 0.39196 & 0.02219 \\ 0.99096 & 0.01036 & 0.30244 \\ 0.93957 & 0.44063 & 0.53621 \\ 0.99241 & 0.01197 & 0.40194 \\ 0.29716 & 0.68823 & 0.01860 \\ 0.39586 & 0.58348 & 0.02292 \\ 0.07652 & 0.92879 & 0.44781 \\ 0.99901 & 0.00962 & 0.69061 \\ 0.99662 & 0.01177 & 0.58933 \\ 0.69085 & 0.01057 & 0.99765 \\ 0.58959 & 0.01271 & 0.99529 \\ 0.01777 & 0.29316 & 0.68768\end{array}$




$\begin{array}{ccc}0.02206 & 0.39240 & 0.58434 \\ 0.44845 & 0.92928 & 0.07541 \\ 0.30298 & 0.01031 & 0.99019 \\ 0.40232 & 0.01241 & 0.99144 \\ 0.01666 & 0.68952 & 0.29784 \\ 0.02127 & 0.58468 & 0.39640 \\ 0.44441 & 0.53552 & 0.10486 \\ 0.08816 & 0.54205 & 0.92108 \\ 0.91289 & 0.09527 & 0.54318 \\ 0.54204 & 0.92925 & 0.07699 \\ 0.10469 & 0.44009 & 0.53767 \\ 0.44967 & 0.09604 & 0.90977 \\ 0.10391 & 0.53651 & 0.44408 \\ 0.93956 & 0.53620 & 0.44217 \\ 0.54313 & 0.09624 & 0.91179 \\ 0.53834 & 0.43947 & 0.10488 \\ 0.07827 & 0.92849 & 0.54150 \\ 0.44118 & 0.53508 & 0.94121 \\ 0.91068 & 0.09528 & 0.44970 \\ 1.49569 & 0.24513 & 0.37167 \\ 1.51032 & -0.14119 & 0.47817 \\ 1.63111 & -0.18346 & 0.35792 \\ 1.53858 & -0.03446 & 0.33665 \\ 1.72333 & -0.06717 & 0.34230 \\ 1.76510 & -0.18687 & 0.52463 \\ 1.64015 & -0.22866 & 0.58592 \\ 1.76134 & -0.27100 & 0.46579 \\ 1.72626 & 0.07241 & 0.52204 \\ 1.67025 & 0.22681 & 0.45719 \\ 1.57519 & 0.15129 & 0.58074 \\ 0.26481 & 0.90214 & 0.67377 \\ 0.67207 & 0.90569 & 0.26276 \\ 0.40918 & 0.86533 & 0.13838 \\ 0.16647 & 0.57661 & 0.40659 \\ 0.58228 & 0.16034 & 0.84897 \\ 0.57856 & 0.40040 & 0.16752 \\ 0.14209 & 0.86388 & 0.58015 \\ 0.40103 & 0.57545 & 0.87826 \\ 0.84622 & 0.15952 & 0.41177 \\ 0.15311 & 0.40886 & 0.85671 \\ 0.85682 & 0.58053 & 0.15240 \\ 0.90476 & 0.28565 & 0.90401 \\ 0.90542 & 0.90821 & 0.90527 \\ 0.29014 & 0.90813 & 0.88704\end{array}$




$\begin{array}{lll}0.88654 & 0.90944 & 0.29025 \\ 0.15206 & 0.58140 & 0.85687 \\ 0.85773 & 0.40787 & 0.15256 \\ 0.57418 & 0.40141 & 0.87635 \\ 0.13948 & 0.86506 & 0.40826 \\ 0.40740 & 0.57546 & 0.16747 \\ 0.85000 & 0.15912 & 0.58263 \\ 0.58083 & 0.86475 & 0.14074 \\ 0.16799 & 0.40081 & 0.57713 \\ 0.41156 & 0.16033 & 0.84544 \\ 0.87602 & 0.57680 & 0.40270 \\ 0.87559 & 0.40193 & 0.57506 \\ 1.58532 & -0.00293 & 0.47553 \\ 1.73817 & 0.11950 & 0.27585 \\ 0.07905 & 0.73299 & 0.92389 \\ 0.26870 & 0.87872 & 0.74720 \\ 0.92529 & 0.26118 & 0.08004 \\ 0.73325 & 0.26180 & 0.93316 \\ 0.07178 & 0.93752 & 0.25844 \\ 0.26692 & 0.73882 & 0.08480 \\ 0.92213 & 0.08668 & 0.73209 \\ 0.72879 & 0.93197 & 0.07539 \\ 0.09790 & 0.24711 & 0.72702 \\ 0.26855 & 0.08237 & 0.90658 \\ 0.94652 & 0.72231 & 0.25318 \\ 0.93376 & 0.26166 & 0.73271 \\ 0.25906 & 0.93751 & 0.07122 \\ 0.08339 & 0.73964 & 0.26712 \\ 0.73270 & 0.08730 & 0.92052 \\ 0.72798 & 0.24740 & 0.09710 \\ 0.07643 & 0.93085 & 0.72894 \\ 0.25191 & 0.72067 & 0.94910 \\ 0.90752 & 0.08275 & 0.26796 \\ 0.08097 & 0.26128 & 0.92414 \\ 0.74517 & 0.88152 & 0.26773 \\ 0.92298 & 0.73310 & 0.07963 \\ 0.05035 & 0.05307 & 0.04982 \\ 0.05238 & 0.05061 & 0.84803 \\ 0.05163 & 0.85524 & 0.05233 \\ 0.84834 & 0.05113 & 0.05171 \\ 0.93283 & 0.93418 & 0.93279 \\ 0.91870 & 0.93804 & 0.20845 \\ 0.20841 & 0.93696 & 0.91960 \\ 0.93275 & 0.20430 & 0.93196\end{array}$




$$
\begin{array}{lll}
1.61695 & 0.12725 & 0.40544 \\
0.12021 & 0.88041 & 0.12012 \\
0.88154 & 0.11945 & 0.88088 \\
0.87743 & 0.87740 & 0.12346 \\
0.11777 & 0.11828 & 0.88270 \\
0.88331 & 0.11860 & 0.11716 \\
0.12343 & 0.87656 & 0.87831
\end{array}
$$

GB at Defect (F orientation):

POSCAR file from uio-66-prim-defect-gb-1-f.res: C: 44 F: 1 H: 36 O: 32 P: 1 ZR: 6 14.6640000000

$\begin{array}{lll}0.8119283518 & 0.2940430090 & 0.5049819426 \\ 0.0000000000 & 0.8679239315 & 0.5037576415 \\ 0.0000000000 & 0.0000000000 & 1.0000000000\end{array}$

\section{6}

Direct

$\begin{array}{lll}0.92177 & 0.44699 & 0.08830 \\ 0.08871 & 0.44750 & 0.92110 \\ 1.44619 & 0.28751 & 0.19721 \\ 1.52112 & 0.17068 & 0.25019 \\ 0.92119 & 0.54156 & 0.08824 \\ 1.48801 & 0.08114 & 0.26046 \\ 1.64830 & 0.00002 & 0.51391 \\ 0.53562 & 0.43993 & 0.94044 \\ 0.00215 & 0.69240 & 0.00286 \\ 0.00409 & 0.58960 & 0.00434 \\ 0.00411 & 0.30036 & 0.00305 \\ 0.00525 & 0.39991 & 0.00447 \\ 0.68830 & 0.29318 & 0.01724 \\ 0.58459 & 0.39196 & 0.02219 \\ 0.99096 & 0.01036 & 0.30244 \\ 0.93957 & 0.44063 & 0.53621 \\ 0.99241 & 0.01197 & 0.40194 \\ 0.29716 & 0.68823 & 0.01860 \\ 0.39586 & 0.58348 & 0.02292 \\ 0.07652 & 0.92879 & 0.44781 \\ 0.99901 & 0.00962 & 0.69061 \\ 0.99662 & 0.01177 & 0.58933 \\ 0.69085 & 0.01057 & 0.99765 \\ 0.58959 & 0.01271 & 0.99529 \\ 0.01777 & 0.29316 & 0.68768 \\ 0.02206 & 0.39240 & 0.58434 \\ 0.44845 & 0.92928 & 0.07541\end{array}$




$\begin{array}{ccc}0.30298 & 0.01031 & 0.99019 \\ 0.40232 & 0.01241 & 0.99144 \\ 0.01666 & 0.68952 & 0.29784 \\ 0.02127 & 0.58468 & 0.39640 \\ 0.44441 & 0.53552 & 0.10486 \\ 0.08816 & 0.54205 & 0.92108 \\ 0.91289 & 0.09527 & 0.54318 \\ 0.54204 & 0.92925 & 0.07699 \\ 0.10469 & 0.44009 & 0.53767 \\ 0.44967 & 0.09604 & 0.90977 \\ 0.10391 & 0.53651 & 0.44408 \\ 0.93956 & 0.53620 & 0.44217 \\ 0.54313 & 0.09624 & 0.91179 \\ 0.53834 & 0.43947 & 0.10488 \\ 0.07827 & 0.92849 & 0.54150 \\ 0.44118 & 0.53508 & 0.94121 \\ 0.91068 & 0.09528 & 0.44970 \\ 1.59542 & 0.24831 & 0.43403 \\ 1.35454 & 0.29896 & 0.25224 \\ 1.45810 & 0.29609 & 0.11053 \\ 1.47054 & 0.35385 & 0.18972 \\ 1.61293 & 0.15904 & 0.19532 \\ 1.54335 & -0.00549 & 0.29990 \\ 1.39620 & 0.09304 & 0.31531 \\ 1.50005 & 0.08965 & 0.17372 \\ 1.66653 & -0.07171 & 0.48997 \\ 1.72263 & -0.01590 & 0.53266 \\ 1.56986 & 0.00616 & 0.59132 \\ 0.26481 & 0.90214 & 0.67377 \\ 0.67207 & 0.90569 & 0.26276 \\ 0.40918 & 0.86533 & 0.13838 \\ 0.16647 & 0.57661 & 0.40659 \\ 0.58228 & 0.16034 & 0.84897 \\ 0.57856 & 0.40040 & 0.16752 \\ 0.14209 & 0.86388 & 0.58015 \\ 0.40103 & 0.57545 & 0.87826 \\ 0.84622 & 0.15952 & 0.41177 \\ 0.15311 & 0.40886 & 0.85671 \\ 0.85682 & 0.58053 & 0.15240 \\ 0.90476 & 0.28565 & 0.90401 \\ 0.90542 & 0.90821 & 0.90527 \\ 0.29014 & 0.90813 & 0.88704 \\ 0.88654 & 0.90944 & 0.29025 \\ 0.15206 & 0.58140 & 0.85687\end{array}$




$\begin{array}{lll}0.85773 & 0.40787 & 0.15256 \\ 0.57418 & 0.40141 & 0.87635 \\ 0.13948 & 0.86506 & 0.40826 \\ 0.40740 & 0.57546 & 0.16747 \\ 0.85000 & 0.15912 & 0.58263 \\ 0.58083 & 0.86475 & 0.14074 \\ 0.16799 & 0.40081 & 0.57713 \\ 0.41156 & 0.16033 & 0.84544 \\ 0.87602 & 0.57680 & 0.40270 \\ 0.87559 & 0.40193 & 0.57506 \\ 1.50503 & 0.15934 & 0.36506 \\ 1.75148 & 0.12439 & 0.27044 \\ 0.07905 & 0.73299 & 0.92389 \\ 0.26870 & 0.87872 & 0.74720 \\ 0.92529 & 0.26118 & 0.08004 \\ 0.73325 & 0.26180 & 0.93316 \\ 0.07178 & 0.93752 & 0.25844 \\ 0.26692 & 0.73882 & 0.08480 \\ 0.92213 & 0.08668 & 0.73209 \\ 0.72879 & 0.93197 & 0.07539 \\ 0.09790 & 0.24711 & 0.72702 \\ 0.26855 & 0.08237 & 0.90658 \\ 0.94652 & 0.72231 & 0.25318 \\ 0.93376 & 0.26166 & 0.73271 \\ 0.25906 & 0.93751 & 0.07122 \\ 0.08339 & 0.73964 & 0.26712 \\ 0.73270 & 0.08730 & 0.92052 \\ 0.72798 & 0.24740 & 0.09710 \\ 0.07643 & 0.93085 & 0.72894 \\ 0.25191 & 0.72067 & 0.94910 \\ 0.90752 & 0.08275 & 0.26796 \\ 0.08097 & 0.26128 & 0.92414 \\ 0.74517 & 0.88152 & 0.26773 \\ 0.92298 & 0.73310 & 0.07963 \\ 0.05035 & 0.05307 & 0.04982 \\ 0.05238 & 0.05061 & 0.84803 \\ 0.05163 & 0.85524 & 0.05233 \\ 0.84834 & 0.05113 & 0.05171 \\ 0.93283 & 0.93418 & 0.93279 \\ 0.91870 & 0.93804 & 0.20845 \\ 0.20841 & 0.93696 & 0.91960 \\ 0.93275 & 0.20430 & 0.93196 \\ 1.62489 & 0.13386 & 0.39549 \\ 0.12021 & 0.88041 & 0.12012\end{array}$




$$
\begin{array}{lll}
0.88154 & 0.11945 & 0.88088 \\
0.87743 & 0.87740 & 0.12346 \\
0.11777 & 0.11828 & 0.88270 \\
0.88331 & 0.11860 & 0.11716 \\
0.12343 & 0.87656 & 0.87831
\end{array}
$$

GB at Defect (O(sp3) orientation):

POSCAR file from uio-66-prim-defect-gb-1-osp3.res: C: 44 F: 1 H: 36 O: 32 P: 1 ZR: 6 14.6640000000

$\begin{array}{lll}0.8119283518 & 0.2940430090 & 0.5049819426 \\ 0.0000000000 & 0.8679239315 & 0.5037576415 \\ 0.0000000000 & 0.0000000000 & 1.0000000000\end{array}$

441363216

Direct

$\begin{array}{lll}0.92177 & 0.44699 & 0.08830 \\ 0.08871 & 0.44750 & 0.92110 \\ 1.63561 & 0.39266 & 0.41661 \\ 1.59485 & 0.36305 & 0.35987 \\ 0.92119 & 0.54156 & 0.08824 \\ 1.49416 & 0.46256 & 0.32708 \\ 1.52772 & 0.09706 & 0.40247 \\ 0.53562 & 0.43993 & 0.94044 \\ 0.00215 & 0.69240 & 0.00286 \\ 0.00409 & 0.58960 & 0.00434 \\ 0.00411 & 0.30036 & 0.00305 \\ 0.00525 & 0.39991 & 0.00447 \\ 0.68830 & 0.29318 & 0.01724 \\ 0.58459 & 0.39196 & 0.02219 \\ 0.99096 & 0.01036 & 0.30244 \\ 0.93957 & 0.44063 & 0.53621 \\ 0.99241 & 0.01197 & 0.40194 \\ 0.29716 & 0.68823 & 0.01860 \\ 0.39586 & 0.58348 & 0.02292 \\ 0.07652 & 0.92879 & 0.44781 \\ 0.99901 & 0.00962 & 0.69061 \\ 0.99662 & 0.01177 & 0.58933 \\ 0.69085 & 0.01057 & 0.99765 \\ 0.58959 & 0.01271 & 0.99529 \\ 0.01777 & 0.29316 & 0.68768 \\ 0.02206 & 0.39240 & 0.58434 \\ 0.44845 & 0.92928 & 0.07541 \\ 0.30298 & 0.01031 & 0.99019 \\ 0.40232 & 0.01241 & 0.99144\end{array}$




$\begin{array}{lll}0.01666 & 0.68952 & 0.29784 \\ 0.02127 & 0.58468 & 0.39640 \\ 0.44441 & 0.53552 & 0.10486 \\ 0.08816 & 0.54205 & 0.92108 \\ 0.91289 & 0.09527 & 0.54318 \\ 0.54204 & 0.92925 & 0.07699 \\ 0.10469 & 0.44009 & 0.53767 \\ 0.44967 & 0.09604 & 0.90977 \\ 0.10391 & 0.53651 & 0.44408 \\ 0.93956 & 0.53620 & 0.44217 \\ 0.54313 & 0.09624 & 0.91179 \\ 0.53834 & 0.43947 & 0.10488 \\ 0.07827 & 0.92849 & 0.54150 \\ 0.44118 & 0.53508 & 0.94121 \\ 0.91068 & 0.09528 & 0.44970 \\ 1.69628 & 0.01965 & 0.50842 \\ 1.56452 & 0.41186 & 0.49469 \\ 1.66237 & 0.46902 & 0.35604 \\ 1.71005 & 0.31899 & 0.44105 \\ 1.66589 & 0.34370 & 0.28174 \\ 1.46374 & 0.44065 & 0.28520 \\ 1.42334 & 0.48171 & 0.40535 \\ 1.52098 & 0.53890 & 0.26640 \\ 1.48693 & 0.16834 & 0.33757 \\ 1.57253 & 0.01744 & 0.38013 \\ 1.46035 & 0.08328 & 0.48862 \\ 0.26481 & 0.90214 & 0.67377 \\ 0.67207 & 0.90569 & 0.26276 \\ 0.40918 & 0.86533 & 0.13838 \\ 0.16647 & 0.57661 & 0.40659 \\ 0.58228 & 0.16034 & 0.84897 \\ 0.57856 & 0.40040 & 0.16752 \\ 0.14209 & 0.86388 & 0.58015 \\ 0.40103 & 0.57545 & 0.87826 \\ 0.84622 & 0.15952 & 0.41177 \\ 0.15311 & 0.40886 & 0.85671 \\ 0.85682 & 0.58053 & 0.15240 \\ 0.90476 & 0.28565 & 0.90401 \\ 0.90542 & 0.90821 & 0.90527 \\ 0.29014 & 0.90813 & 0.88704 \\ 0.88654 & 0.90944 & 0.29025 \\ 0.15206 & 0.58140 & 0.85687 \\ 0.85773 & 0.40787 & 0.15256 \\ 0.57418 & 0.40141 & 0.87635\end{array}$




$\begin{array}{lll}0.13948 & 0.86506 & 0.40826 \\ 0.40740 & 0.57546 & 0.16747 \\ 0.85000 & 0.15912 & 0.58263 \\ 0.58083 & 0.86475 & 0.14074 \\ 0.16799 & 0.40081 & 0.57713 \\ 0.41156 & 0.16033 & 0.84544 \\ 0.87602 & 0.57680 & 0.40270 \\ 0.87559 & 0.40193 & 0.57506 \\ 1.55916 & 0.26215 & 0.44026 \\ 1.74065 & 0.15539 & 0.26562 \\ 0.07905 & 0.73299 & 0.92389 \\ 0.26870 & 0.87872 & 0.74720 \\ 0.92529 & 0.26118 & 0.08004 \\ 0.73325 & 0.26180 & 0.93316 \\ 0.07178 & 0.93752 & 0.25844 \\ 0.26692 & 0.73882 & 0.08480 \\ 0.92213 & 0.08668 & 0.73209 \\ 0.72879 & 0.93197 & 0.07539 \\ 0.09790 & 0.24711 & 0.72702 \\ 0.26855 & 0.08237 & 0.90658 \\ 0.94652 & 0.72231 & 0.25318 \\ 0.93376 & 0.26166 & 0.73271 \\ 0.25906 & 0.93751 & 0.07122 \\ 0.08339 & 0.73964 & 0.26712 \\ 0.73270 & 0.08730 & 0.92052 \\ 0.72798 & 0.24740 & 0.09710 \\ 0.07643 & 0.93085 & 0.72894 \\ 0.25191 & 0.72067 & 0.94910 \\ 0.90752 & 0.08275 & 0.26796 \\ 0.08097 & 0.26128 & 0.92414 \\ 0.74517 & 0.88152 & 0.26773 \\ 0.92298 & 0.73310 & 0.07963 \\ 0.05035 & 0.05307 & 0.04982 \\ 0.05238 & 0.05061 & 0.84803 \\ 0.05163 & 0.85524 & 0.05233 \\ 0.84834 & 0.05113 & 0.05171 \\ 0.93283 & 0.93418 & 0.93279 \\ 0.91870 & 0.93804 & 0.20845 \\ 0.20841 & 0.93696 & 0.91960 \\ 0.93275 & 0.20430 & 0.93196 \\ 1.63155 & 0.13339 & 0.40448 \\ 0.12021 & 0.88041 & 0.12012 \\ 0.88154 & 0.11945 & 0.88088 \\ 0.87743 & 0.87740 & 0.12346\end{array}$




$\begin{array}{lll}0.11777 & 0.11828 & 0.88270 \\ 0.88331 & 0.11860 & 0.11716 \\ 0.12343 & 0.87656 & 0.87831\end{array}$

GB at Ideal:

POSCAR file from uio-66-prim-defect-gb-ideal-site.res: C: $44 \mathrm{~F}: 1 \mathrm{H}: 36$ O: 32 P: 1 ZR: 6 14.6640000000

$\begin{array}{lll}0.8119283518 & 0.2940430090 & 0.5049819426 \\ 0.0000000000 & 0.8679239315 & 0.5037576415 \\ 0.0000000000 & 0.0000000000 & 1.0000000000\end{array}$

441363216

Direct

$\begin{array}{lll}0.92177 & 0.44699 & 0.08830 \\ 0.08871 & 0.44750 & 0.92110 \\ 1.22761 & 0.92590 & 0.22206 \\ 1.27976 & 0.88154 & 0.31130 \\ 0.92119 & 0.54156 & 0.08824 \\ 1.35079 & 0.95107 & 0.27363 \\ 1.30544 & 0.55724 & 0.38421 \\ 0.53562 & 0.43993 & 0.94044 \\ 0.00215 & 0.69240 & 0.00286 \\ 0.00409 & 0.58960 & 0.00434 \\ 0.00411 & 0.30036 & 0.00305 \\ 0.00525 & 0.39991 & 0.00447 \\ 0.68830 & 0.29318 & 0.01724 \\ 0.58459 & 0.39196 & 0.02219 \\ 0.99096 & 0.01036 & 0.30244 \\ 0.93957 & 0.44063 & 0.53621 \\ 0.99241 & 0.01197 & 0.40194 \\ 0.29716 & 0.68823 & 0.01860 \\ 0.39586 & 0.58348 & 0.02292 \\ 0.07652 & 0.92879 & 0.44781 \\ 0.99901 & 0.00962 & 0.69061 \\ 0.99662 & 0.01177 & 0.58933 \\ 0.69085 & 0.01057 & 0.99765 \\ 0.58959 & 0.01271 & 0.99529 \\ 0.01777 & 0.29316 & 0.68768 \\ 0.02206 & 0.39240 & 0.58434 \\ 0.44845 & 0.92928 & 0.07541 \\ 0.30298 & 0.01031 & 0.99019 \\ 0.40232 & 0.01241 & 0.99144 \\ 0.01666 & 0.68952 & 0.29784 \\ 0.02127 & 0.58468 & 0.39640\end{array}$




$\begin{array}{lll}0.44441 & 0.53552 & 0.10486 \\ 0.08816 & 0.54205 & 0.92108 \\ 0.91289 & 0.09527 & 0.54318 \\ 0.54204 & 0.92925 & 0.07699 \\ 0.10469 & 0.44009 & 0.53767 \\ 0.44967 & 0.09604 & 0.90977 \\ 0.10391 & 0.53651 & 0.44408 \\ 0.93956 & 0.53620 & 0.44217 \\ 0.54313 & 0.09624 & 0.91179 \\ 0.53834 & 0.43947 & 0.10488 \\ 0.07827 & 0.92849 & 0.54150 \\ 0.44118 & 0.53508 & 0.94121 \\ 0.91068 & 0.09528 & 0.44970 \\ 1.47759 & 0.63463 & 0.16471 \\ 1.29735 & 0.91897 & 0.13912 \\ 1.17181 & 1.01714 & 0.21126 \\ 1.17514 & 0.87439 & 0.24979 \\ 1.21013 & 0.88830 & 0.39443 \\ 1.38965 & 0.91814 & 0.33961 \\ 1.42026 & 0.94418 & 0.19050 \\ 1.29494 & 1.04231 & 0.26296 \\ 1.22147 & 0.58639 & 0.44926 \\ 1.29871 & 0.50810 & 0.34900 \\ 1.37320 & 0.50157 & 0.42505 \\ 0.26481 & 0.90214 & 0.67377 \\ 0.67207 & 0.90569 & 0.26276 \\ 0.40918 & 0.86533 & 0.13838 \\ 0.16647 & 0.57661 & 0.40659 \\ 0.58228 & 0.16034 & 0.84897 \\ 0.57856 & 0.40040 & 0.16752 \\ 0.14209 & 0.86388 & 0.58015 \\ 0.40103 & 0.57545 & 0.87826 \\ 0.84622 & 0.15952 & 0.41177 \\ 0.15311 & 0.40886 & 0.85671 \\ 0.85682 & 0.58053 & 0.15240 \\ 0.90476 & 0.28565 & 0.90401 \\ 0.90542 & 0.90821 & 0.90527 \\ 0.29014 & 0.90813 & 0.88704 \\ 0.88654 & 0.90944 & 0.29025 \\ 0.15206 & 0.58140 & 0.85687 \\ 0.85773 & 0.40787 & 0.15256 \\ 0.57418 & 0.40141 & 0.87635 \\ 0.13948 & 0.86506 & 0.40826 \\ 0.40740 & 0.57546 & 0.16747\end{array}$




$\begin{array}{lll}0.85000 & 0.15912 & 0.58263 \\ 0.58083 & 0.86475 & 0.14074 \\ 0.16799 & 0.40081 & 0.57713 \\ 0.41156 & 0.16033 & 0.84544 \\ 0.87602 & 0.57680 & 0.40270 \\ 0.87559 & 0.40193 & 0.57506 \\ 1.35385 & 0.76084 & 0.32527 \\ 1.23426 & 0.77130 & 0.20191 \\ 0.07905 & 0.73299 & 0.92389 \\ 0.26870 & 0.87872 & 0.74720 \\ 0.92529 & 0.26118 & 0.08004 \\ 0.73325 & 0.26180 & 0.93316 \\ 0.07178 & 0.93752 & 0.25844 \\ 0.26692 & 0.73882 & 0.08480 \\ 0.92213 & 0.08668 & 0.73209 \\ 0.72879 & 0.93197 & 0.07539 \\ 0.09790 & 0.24711 & 0.72702 \\ 0.26855 & 0.08237 & 0.90658 \\ 0.94652 & 0.72231 & 0.25318 \\ 0.93376 & 0.26166 & 0.73271 \\ 0.25906 & 0.93751 & 0.07122 \\ 0.08339 & 0.73964 & 0.26712 \\ 0.73270 & 0.08730 & 0.92052 \\ 0.72798 & 0.24740 & 0.09710 \\ 0.07643 & 0.93085 & 0.72894 \\ 0.25191 & 0.72067 & 0.94910 \\ 0.90752 & 0.08275 & 0.26796 \\ 0.08097 & 0.26128 & 0.92414 \\ 0.74517 & 0.88152 & 0.26773 \\ 0.92298 & 0.73310 & 0.07963 \\ 0.05035 & 0.05307 & 0.04982 \\ 0.05238 & 0.05061 & 0.84803 \\ 0.05163 & 0.85524 & 0.05233 \\ 0.84834 & 0.05113 & 0.05171 \\ 0.93283 & 0.93418 & 0.93279 \\ 0.91870 & 0.93804 & 0.20845 \\ 0.20841 & 0.93696 & 0.91960 \\ 0.93275 & 0.20430 & 0.93196 \\ 1.34330 & 0.68137 & 0.26827 \\ 0.12021 & 0.88041 & 0.12012 \\ 0.88154 & 0.11945 & 0.88088 \\ 0.87743 & 0.87740 & 0.12346 \\ 0.11777 & 0.11828 & 0.88270 \\ 0.88331 & 0.11860 & 0.11716\end{array}$




\section{$0.12343 \quad 0.87656 \quad 0.87831$}

$\mathrm{GB}$ at $\mathrm{ZrOH}$ :

POSCAR file from uio-66-prim-defect-gb-zroh.res: C: 44 F: 1 H: 36 O: 32 P: 1 ZR: 6 14.6640000000

$\begin{array}{lll}0.8119283518 & 0.2940430090 & 0.5049819426 \\ 0.0000000000 & 0.8679239315 & 0.5037576415 \\ 0.0000000000 & 0.0000000000 & 1.0000000000\end{array}$

441363216

Direct

$\begin{array}{lll}0.92177 & 0.44699 & 0.08830 \\ 0.08871 & 0.44750 & 0.92110 \\ 1.41790 & 0.16782 & 0.45128 \\ 1.39742 & 0.13604 & 0.37956 \\ 0.92119 & 0.54156 & 0.08824 \\ 1.31867 & 0.23998 & 0.31754 \\ 1.32525 & -0.11824 & 0.40441 \\ 0.53562 & 0.43993 & 0.94044 \\ 0.00215 & 0.69240 & 0.00286 \\ 0.00409 & 0.58960 & 0.00434 \\ 0.00411 & 0.30036 & 0.00305 \\ 0.00525 & 0.39991 & 0.00447 \\ 0.68830 & 0.29318 & 0.01724 \\ 0.58459 & 0.39196 & 0.02219 \\ 0.99096 & 0.01036 & 0.30244 \\ 0.93957 & 0.44063 & 0.53621 \\ 0.99241 & 0.01197 & 0.40194 \\ 0.29716 & 0.68823 & 0.01860 \\ 0.39586 & 0.58348 & 0.02292 \\ 0.07652 & 0.92879 & 0.44781 \\ 0.99901 & 0.00962 & 0.69061 \\ 0.99662 & 0.01177 & 0.58933 \\ 0.69085 & 0.01057 & 0.99765 \\ 0.58959 & 0.01271 & 0.99529 \\ 0.01777 & 0.29316 & 0.68768 \\ 0.02206 & 0.39240 & 0.58434 \\ 0.44845 & 0.92928 & 0.07541 \\ 0.30298 & 0.01031 & 0.99019 \\ 0.40232 & 0.01241 & 0.99144 \\ 0.01666 & 0.68952 & 0.29784 \\ 0.02127 & 0.58468 & 0.39640 \\ 0.44441 & 0.53552 & 0.10486 \\ 0.08816 & 0.54205 & 0.92108\end{array}$




$\begin{array}{lrl}0.91289 & 0.09527 & 0.54318 \\ 0.54204 & 0.92925 & 0.07699 \\ 0.10469 & 0.44009 & 0.53767 \\ 0.44967 & 0.09604 & 0.90977 \\ 0.10391 & 0.53651 & 0.44408 \\ 0.93956 & 0.53620 & 0.44217 \\ 0.54313 & 0.09624 & 0.91179 \\ 0.53834 & 0.43947 & 0.10488 \\ 0.07827 & 0.92849 & 0.54150 \\ 0.44118 & 0.53508 & 0.94121 \\ 0.91068 & 0.09528 & 0.44970 \\ 1.44373 & -0.19844 & 0.56304 \\ 1.33441 & 0.20080 & 0.51459 \\ 1.45835 & 0.23527 & 0.39491 \\ 1.47606 & 0.09090 & 0.49734 \\ 1.48088 & 0.10291 & 0.31618 \\ 1.30326 & 0.21649 & 0.26451 \\ 1.23536 & 0.27290 & 0.38112 \\ 1.35921 & 0.30738 & 0.26107 \\ 1.30761 & -0.05063 & 0.32470 \\ 1.37013 & -0.20336 & 0.39258 \\ 1.24261 & -0.11751 & 0.47710 \\ 0.26481 & 0.90214 & 0.67377 \\ 0.67207 & 0.90569 & 0.26276 \\ 0.40918 & 0.86533 & 0.13838 \\ 0.16647 & 0.57661 & 0.40659 \\ 0.58228 & 0.16034 & 0.84897 \\ 0.57856 & 0.40040 & 0.16752 \\ 0.14209 & 0.86388 & 0.58015 \\ 0.40103 & 0.57545 & 0.87826 \\ 0.84622 & 0.15952 & 0.41177 \\ 0.15311 & 0.40886 & 0.85671 \\ 0.85682 & 0.58053 & 0.15240 \\ 0.90476 & 0.28565 & 0.90401 \\ 0.90542 & 0.90821 & 0.90527 \\ 0.29014 & 0.90813 & 0.88704 \\ 0.88654 & 0.90944 & 0.29025 \\ 0.15206 & 0.58140 & 0.85687 \\ 0.85773 & 0.40787 & 0.15256 \\ 0.57418 & 0.40141 & 0.87635 \\ 0.13948 & 0.86506 & 0.40826 \\ 0.40740 & 0.57546 & 0.16747 \\ 0.85000 & 0.15912 & 0.58263 \\ 0.58083 & 0.86475 & 0.14074\end{array}$




$\begin{array}{lcc}0.16799 & 0.40081 & 0.57713 \\ 0.41156 & 0.16033 & 0.84544 \\ 0.87602 & 0.57680 & 0.40270 \\ 0.87559 & 0.40193 & 0.57506 \\ 1.34358 & 0.04698 & 0.45433 \\ 1.54964 & -0.09206 & 0.31852 \\ 0.07905 & 0.73299 & 0.92389 \\ 0.26870 & 0.87872 & 0.74720 \\ 0.92529 & 0.26118 & 0.08004 \\ 0.73325 & 0.26180 & 0.93316 \\ 0.07178 & 0.93752 & 0.25844 \\ 0.26692 & 0.73882 & 0.08480 \\ 0.92213 & 0.08668 & 0.73209 \\ 0.72879 & 0.93197 & 0.07539 \\ 0.09790 & 0.24711 & 0.72702 \\ 0.26855 & 0.08237 & 0.90658 \\ 0.94652 & 0.72231 & 0.25318 \\ 0.93376 & 0.26166 & 0.73271 \\ 0.25906 & 0.93751 & 0.07122 \\ 0.08339 & 0.73964 & 0.26712 \\ 0.73270 & 0.08730 & 0.92052 \\ 0.72798 & 0.24740 & 0.09710 \\ 0.07643 & 0.93085 & 0.72894 \\ 0.25191 & 0.72067 & 0.94910 \\ 0.90752 & 0.08275 & 0.26796 \\ 0.08097 & 0.26128 & 0.92414 \\ 0.74517 & 0.88152 & 0.26773 \\ 0.92298 & 0.73310 & 0.07963 \\ 0.05035 & 0.05307 & 0.04982 \\ 0.05238 & 0.05061 & 0.84803 \\ 0.05163 & 0.85524 & 0.05233 \\ 0.84834 & 0.05113 & 0.05171 \\ 0.93283 & 0.93418 & 0.93279 \\ 0.91870 & 0.93804 & 0.20845 \\ 0.20841 & 0.93696 & 0.91960 \\ 0.93275 & 0.20430 & 0.93196 \\ 1.41600 & -0.09063 & 0.43554 \\ 0.12021 & 0.88041 & 0.12012 \\ 0.88154 & 0.11945 & 0.88088 \\ 0.87743 & 0.87740 & 0.12346 \\ 0.11777 & 0.11828 & 0.88270 \\ 0.11860 & 0.11716 \\ 0.833 & 0.87656 & 0.87831\end{array}$


$\mathrm{GB}$ at $\mu 3-\mathrm{OH}$ (C orientation):

POSCAR file from uio-66-prim-defect-gb-u3oh-c.res: C: 44 F: 1 H: 36 O: 32 P: 1 ZR: 6 14.6640000000

$\begin{array}{lll}0.8119283518 & 0.2940430090 & 0.5049819426 \\ 0.0000000000 & 0.8679239315 & 0.5037576415 \\ 0.0000000000 & 0.0000000000 & 1.0000000000\end{array}$

441363216

Direct

$\begin{array}{lrr}0.92177 & 0.44699 & 0.08830 \\ 0.08871 & 0.44750 & 0.92110 \\ 1.78353 & -0.46012 & 0.65688 \\ 1.79877 & -0.35240 & 0.63057 \\ 0.92119 & 0.54156 & 0.08824 \\ 1.84132 & -0.37039 & 0.71726 \\ 1.62976 & -0.00678 & 0.54127 \\ 0.53562 & 0.43993 & 0.94044 \\ 0.00215 & 0.69240 & 0.00286 \\ 0.00409 & 0.58960 & 0.00434 \\ 0.00411 & 0.30036 & 0.00305 \\ 0.00525 & 0.39991 & 0.00447 \\ 0.68830 & 0.29318 & 0.01724 \\ 0.58459 & 0.39196 & 0.02219 \\ 0.99096 & 0.01036 & 0.30244 \\ 0.93957 & 0.44063 & 0.53621 \\ 0.99241 & 0.01197 & 0.40194 \\ 0.29716 & 0.68823 & 0.01860 \\ 0.39586 & 0.58348 & 0.02292 \\ 0.07652 & 0.92879 & 0.44781 \\ 0.99901 & 0.00962 & 0.69061 \\ 0.99662 & 0.01177 & 0.58933 \\ 0.69085 & 0.01057 & 0.99765 \\ 0.58959 & 0.01271 & 0.99529 \\ 0.01777 & 0.29316 & 0.68768 \\ 0.02206 & 0.39240 & 0.58434 \\ 0.44845 & 0.92928 & 0.07541 \\ 0.30298 & 0.01031 & 0.99019 \\ 0.40232 & 0.01241 & 0.99144 \\ 0.01666 & 0.68952 & 0.29784 \\ 0.02127 & 0.58468 & 0.39640 \\ 0.44441 & 0.53552 & 0.10486 \\ 0.08816 & 0.54205 & 0.92108 \\ 0.91289 & 0.09527 & 0.54318 \\ 0.54204 & 0.92925 & 0.07699\end{array}$




$\begin{array}{lll}0.10469 & 0.44009 & 0.53767 \\ 0.44967 & 0.09604 & 0.90977 \\ 0.10391 & 0.53651 & 0.44408 \\ 0.93956 & 0.53620 & 0.44217 \\ 0.54313 & 0.09624 & 0.91179 \\ 0.53834 & 0.43947 & 0.10488 \\ 0.07827 & 0.92849 & 0.54150 \\ 0.44118 & 0.53508 & 0.94121 \\ 0.91068 & 0.09528 & 0.44970 \\ 1.56411 & -0.12033 & 0.47214 \\ 1.71995 & -0.48162 & 0.74429 \\ 1.86748 & -0.53153 & 0.65297 \\ 1.75189 & -0.44684 & 0.59284 \\ 1.86230 & -0.33065 & 0.54307 \\ 1.85245 & -0.29054 & 0.69798 \\ 1.77765 & -0.39217 & 0.80461 \\ 1.92535 & -0.44174 & 0.71326 \\ 1.69700 & -0.01556 & 0.56572 \\ 1.62018 & 0.06981 & 0.46365 \\ 1.54638 & 0.00310 & 0.61314 \\ 0.26481 & 0.90214 & 0.67377 \\ 0.67207 & 0.90569 & 0.26276 \\ 0.40918 & 0.86533 & 0.13838 \\ 0.16647 & 0.57661 & 0.40659 \\ 0.58228 & 0.16034 & 0.84897 \\ 0.57856 & 0.40040 & 0.16752 \\ 0.14209 & 0.86388 & 0.58015 \\ 0.40103 & 0.57545 & 0.87826 \\ 0.84622 & 0.15952 & 0.41177 \\ 0.15311 & 0.40886 & 0.85671 \\ 0.85682 & 0.58053 & 0.15240 \\ 0.90476 & 0.28565 & 0.90401 \\ 0.90542 & 0.90821 & 0.90527 \\ 0.29014 & 0.90813 & 0.88704 \\ 0.88654 & 0.90944 & 0.29025 \\ 0.15206 & 0.58140 & 0.85687 \\ 0.85773 & 0.40787 & 0.15256 \\ 0.57418 & 0.40141 & 0.87635 \\ 0.13948 & 0.86506 & 0.40826 \\ 0.40740 & 0.57546 & 0.16747 \\ 0.85000 & 0.15912 & 0.58263 \\ 0.58083 & 0.86475 & 0.14074 \\ 0.40081 & 0.57713 \\ 0.16033 & 0.84544\end{array}$




$\begin{array}{lrl}0.87602 & 0.57680 & 0.40270 \\ 0.87559 & 0.40193 & 0.57506 \\ 1.68751 & -0.25803 & 0.63608 \\ 1.80618 & -0.15043 & 0.39457 \\ 0.07905 & 0.73299 & 0.92389 \\ 0.26870 & 0.87872 & 0.74720 \\ 0.92529 & 0.26118 & 0.08004 \\ 0.73325 & 0.26180 & 0.93316 \\ 0.07178 & 0.93752 & 0.25844 \\ 0.26692 & 0.73882 & 0.08480 \\ 0.92213 & 0.08668 & 0.73209 \\ 0.72879 & 0.93197 & 0.07539 \\ 0.09790 & 0.24711 & 0.72702 \\ 0.26855 & 0.08237 & 0.90658 \\ 0.94652 & 0.72231 & 0.25318 \\ 0.93376 & 0.26166 & 0.73271 \\ 0.25906 & 0.93751 & 0.07122 \\ 0.08339 & 0.73964 & 0.26712 \\ 0.73270 & 0.08730 & 0.92052 \\ 0.72798 & 0.24740 & 0.09710 \\ 0.07643 & 0.93085 & 0.72894 \\ 0.25191 & 0.72067 & 0.94910 \\ 0.90752 & 0.08275 & 0.26796 \\ 0.08097 & 0.26128 & 0.92414 \\ 0.74517 & 0.88152 & 0.26773 \\ 0.92298 & 0.73310 & 0.07963 \\ 0.05035 & 0.05307 & 0.04982 \\ 0.05238 & 0.05061 & 0.84803 \\ 0.05163 & 0.85524 & 0.05233 \\ 0.84834 & 0.05113 & 0.05171 \\ 0.93283 & 0.93418 & 0.93279 \\ 0.91870 & 0.93804 & 0.20845 \\ 0.20841 & 0.93696 & 0.91960 \\ 0.93275 & 0.20430 & 0.93196 \\ 1.67176 & -0.13436 & 0.51078 \\ 0.12021 & 0.88041 & 0.12012 \\ 0.88154 & 0.11945 & 0.88088 \\ 0.87743 & 0.87740 & 0.12346 \\ 0.11777 & 0.11828 & 0.88270 \\ 0.88331 & 0.11860 & 0.11716 \\ 0.12343 & 0.87656 & 0.87831\end{array}$

$\mathrm{GB}$ at $\mu 3-\mathrm{OH}$ (F orientation): 
POSCAR file from uio-66-prim-defect-gb-u3oh-f.res: C: 44 F: 1 H: 36 O: 32 P: 1 ZR: 6 14.6640000000

$\begin{array}{lll}0.8119283518 & 0.2940430090 & 0.5049819426 \\ 0.0000000000 & 0.8679239315 & 0.5037576415 \\ 0.0000000000 & 0.0000000000 & 1.0000000000\end{array}$

441363216

Direct

$\begin{array}{rrr}0.92177 & 0.44699 & 0.08830 \\ 0.08871 & 0.44750 & 0.92110 \\ 1.48659 & -0.02745 & 0.39665 \\ 1.57667 & -0.12592 & 0.44761 \\ 0.92119 & 0.54156 & 0.08824 \\ 1.56467 & -0.23654 & 0.47738 \\ 1.71881 & -0.21833 & 0.68061 \\ 0.53562 & 0.43993 & 0.94044 \\ 0.00215 & 0.69240 & 0.00286 \\ 0.00409 & 0.58960 & 0.00434 \\ 0.00411 & 0.30036 & 0.00305 \\ 0.00525 & 0.39991 & 0.00447 \\ 0.68830 & 0.29318 & 0.01724 \\ 0.58459 & 0.39196 & 0.02219 \\ 0.99096 & 0.01036 & 0.30244 \\ 0.93957 & 0.44063 & 0.53621 \\ 0.99241 & 0.01197 & 0.40194 \\ 0.29716 & 0.68823 & 0.01860 \\ 0.39586 & 0.58348 & 0.02292 \\ 0.07652 & 0.92879 & 0.44781 \\ 0.99901 & 0.00962 & 0.69061 \\ 0.99662 & 0.01177 & 0.58933 \\ 0.69085 & 0.01057 & 0.99765 \\ 0.58959 & 0.01271 & 0.99529 \\ 0.01777 & 0.29316 & 0.68768 \\ 0.02206 & 0.39240 & 0.58434 \\ 0.44845 & 0.92928 & 0.07541 \\ 0.30298 & 0.01031 & 0.99019 \\ 0.40232 & 0.01241 & 0.99144 \\ 0.01666 & 0.68952 & 0.29784 \\ 0.02127 & 0.58468 & 0.39640 \\ 0.44441 & 0.53552 & 0.10486 \\ 0.08816 & 0.54205 & 0.92108 \\ 0.91289 & 0.09527 & 0.54318 \\ 0.54204 & 0.92925 & 0.07699 \\ 0.10469 & 0.44009 & 0.53767 \\ 0.44967 & 0.09604 & 0.90977\end{array}$




$\begin{array}{lrl}0.10391 & 0.53651 & 0.44408 \\ 0.93956 & 0.53620 & 0.44217 \\ 0.54313 & 0.09624 & 0.91179 \\ 0.53834 & 0.43947 & 0.10488 \\ 0.07827 & 0.92849 & 0.54150 \\ 0.44118 & 0.53508 & 0.94121 \\ 0.91068 & 0.09528 & 0.44970 \\ 1.62265 & 0.03152 & 0.58067 \\ 1.39969 & -0.02871 & 0.45987 \\ 1.49817 & -0.03535 & 0.31769 \\ 1.49531 & 0.05448 & 0.37473 \\ 1.66374 & -0.12479 & 0.38452 \\ 1.63126 & -0.30958 & 0.51535 \\ 1.47755 & -0.23737 & 0.54040 \\ 1.57638 & -0.24448 & 0.39836 \\ 1.75064 & -0.30121 & 0.66808 \\ 1.78877 & -0.20930 & 0.68549 \\ 1.64428 & -0.21612 & 0.76165 \\ 0.26481 & 0.90214 & 0.67377 \\ 0.67207 & 0.90569 & 0.26276 \\ 0.40918 & 0.86533 & 0.13838 \\ 0.16647 & 0.57661 & 0.40659 \\ 0.58228 & 0.16034 & 0.84897 \\ 0.57856 & 0.40040 & 0.16752 \\ 0.14209 & 0.86388 & 0.58015 \\ 0.40103 & 0.57545 & 0.87826 \\ 0.84622 & 0.15952 & 0.41177 \\ 0.15311 & 0.40886 & 0.85671 \\ 0.85682 & 0.58053 & 0.15240 \\ 0.90476 & 0.28565 & 0.90401 \\ 0.90542 & 0.90821 & 0.90527 \\ 0.29014 & 0.90813 & 0.88704 \\ 0.88654 & 0.90944 & 0.29025 \\ 0.15206 & 0.58140 & 0.85687 \\ 0.85773 & 0.40787 & 0.15256 \\ 0.57418 & 0.40141 & 0.87635 \\ 0.13948 & 0.86506 & 0.40826 \\ 0.40740 & 0.57546 & 0.16747 \\ 0.85000 & 0.15912 & 0.58263 \\ 0.58083 & 0.86475 & 0.14074 \\ 0.16799 & 0.40081 & 0.57713 \\ 0.81156 & 0.16033 & 0.84544 \\ 0.87559 & 0.40193 & 0.57506\end{array}$




$\begin{array}{lll}1.56103 & -0.11552 & 0.55230 \\ 1.79398 & -0.10377 & 0.42932 \\ 0.07905 & 0.73299 & 0.92389 \\ 0.26870 & 0.87872 & 0.74720 \\ 0.92529 & 0.26118 & 0.08004 \\ 0.73325 & 0.26180 & 0.93316 \\ 0.07178 & 0.93752 & 0.25844 \\ 0.26692 & 0.73882 & 0.08480 \\ 0.92213 & 0.08668 & 0.73209 \\ 0.72879 & 0.93197 & 0.07539 \\ 0.09790 & 0.24711 & 0.72702 \\ 0.26855 & 0.08237 & 0.90658 \\ 0.94652 & 0.72231 & 0.25318 \\ 0.93376 & 0.26166 & 0.73271 \\ 0.25906 & 0.93751 & 0.07122 \\ 0.08339 & 0.73964 & 0.26712 \\ 0.73270 & 0.08730 & 0.92052 \\ 0.72798 & 0.24740 & 0.09710 \\ 0.07643 & 0.93085 & 0.72894 \\ 0.25191 & 0.72067 & 0.94910 \\ 0.90752 & 0.08275 & 0.26796 \\ 0.08097 & 0.26128 & 0.92414 \\ 0.74517 & 0.88152 & 0.26773 \\ 0.92298 & 0.73310 & 0.07963 \\ 0.05035 & 0.05307 & 0.04982 \\ 0.05238 & 0.05061 & 0.84803 \\ 0.05163 & 0.85524 & 0.05233 \\ 0.84834 & 0.05113 & 0.05171 \\ 0.93283 & 0.93418 & 0.93279 \\ 0.91870 & 0.93804 & 0.20845 \\ 0.20841 & 0.93696 & 0.91960 \\ 0.93275 & 0.20430 & 0.93196 \\ 1.67381 & -0.10069 & 0.56030 \\ 0.12021 & 0.88041 & 0.12012 \\ 0.88154 & 0.11945 & 0.88088 \\ 0.87743 & 0.87740 & 0.12346 \\ 0.11777 & 0.11828 & 0.88270 \\ 0.88331 & 0.11860 & 0.11716 \\ 0.12343 & 0.87656 & 0.87831\end{array}$

$\mathrm{GB}$ at $\mu 3-\mathrm{OH}(\mathrm{O}(\mathrm{sp} 3)$ orientation):

POSCAR file from uio-66-prim-defect-gb-u3oh.res: C: 44 F: 1 H: 36 O: 32 P: 1 ZR: 6 14.6640000000 


$\begin{array}{lll}0.8119283518 & 0.2940430090 & 0.5049819426 \\ 0.0000000000 & 0.8679239315 & 0.5037576415 \\ 0.0000000000 & 0.0000000000 & 1.0000000000\end{array}$

\section{6}

Direct

$\begin{array}{lll}0.92177 & 0.44699 & 0.08830 \\ 0.08871 & 0.44750 & 0.92110 \\ 1.74648 & 0.09912 & 0.57986 \\ 1.70572 & 0.06951 & 0.52312 \\ 0.92119 & 0.54156 & 0.08824 \\ 1.60503 & 0.16902 & 0.49033 \\ 1.63859 & -0.19648 & 0.56572 \\ 0.53562 & 0.43993 & 0.94044 \\ 0.00215 & 0.69240 & 0.00286 \\ 0.00409 & 0.58960 & 0.00434 \\ 0.00411 & 0.30036 & 0.00305 \\ 0.00525 & 0.39991 & 0.00447 \\ 0.68830 & 0.29318 & 0.01724 \\ 0.58459 & 0.39196 & 0.02219 \\ 0.99096 & 0.01036 & 0.30244 \\ 0.93957 & 0.44063 & 0.53621 \\ 0.99241 & 0.01197 & 0.40194 \\ 0.29716 & 0.68823 & 0.01860 \\ 0.39586 & 0.58348 & 0.02292 \\ 0.07652 & 0.92879 & 0.44781 \\ 0.99901 & 0.00962 & 0.69061 \\ 0.99662 & 0.01177 & 0.58933 \\ 0.69085 & 0.01057 & 0.99765 \\ 0.58959 & 0.01271 & 0.99529 \\ 0.01777 & 0.29316 & 0.68768 \\ 0.02206 & 0.39240 & 0.58434 \\ 0.44845 & 0.92928 & 0.07541 \\ 0.30298 & 0.01031 & 0.99019 \\ 0.40232 & 0.01241 & 0.99144 \\ 0.01666 & 0.68952 & 0.29784 \\ 0.02127 & 0.58468 & 0.39640 \\ 0.44441 & 0.53552 & 0.10486 \\ 0.08816 & 0.54205 & 0.92108 \\ 0.91289 & 0.09527 & 0.54318 \\ 0.54204 & 0.92925 & 0.07699 \\ 0.10469 & 0.44009 & 0.53767 \\ 0.44967 & 0.09604 & 0.90977 \\ 0.10391 & 0.53651 & 0.44408 \\ 0.93956 & 0.53620 & 0.44217\end{array}$




$\begin{array}{rrr}0.54313 & 0.09624 & 0.91179 \\ 0.53834 & 0.43947 & 0.10488 \\ 0.07827 & 0.92849 & 0.54150 \\ 0.44118 & 0.53508 & 0.94121 \\ 0.91068 & 0.09528 & 0.44970 \\ 1.80715 & -0.27389 & 0.67167 \\ 1.67539 & 0.11832 & 0.65794 \\ 1.77324 & 0.17548 & 0.51929 \\ 1.82092 & 0.02545 & 0.60430 \\ 1.77676 & 0.05016 & 0.44499 \\ 1.57461 & 0.14711 & 0.44845 \\ 1.53421 & 0.18817 & 0.56860 \\ 1.63185 & 0.24536 & 0.42965 \\ 1.59780 & -0.12520 & 0.50082 \\ 1.68340 & -0.27610 & 0.54338 \\ 1.57122 & -0.21026 & 0.65187 \\ 0.26481 & 0.90214 & 0.67377 \\ 0.67207 & 0.90569 & 0.26276 \\ 0.40918 & 0.86533 & 0.13838 \\ 0.16647 & 0.57661 & 0.40659 \\ 0.58228 & 0.16034 & 0.84897 \\ 0.57856 & 0.40040 & 0.16752 \\ 0.14209 & 0.86388 & 0.58015 \\ 0.40103 & 0.57545 & 0.87826 \\ 0.84622 & 0.15952 & 0.41177 \\ 0.15311 & 0.40886 & 0.85671 \\ 0.85682 & 0.58053 & 0.15240 \\ 0.90476 & 0.28565 & 0.90401 \\ 0.90542 & 0.90821 & 0.90527 \\ 0.29014 & 0.90813 & 0.88704 \\ 0.88654 & 0.90944 & 0.29025 \\ 0.15206 & 0.58140 & 0.85687 \\ 0.85773 & 0.40787 & 0.15256 \\ 0.57418 & 0.40141 & 0.87635 \\ 0.13948 & 0.86506 & 0.40826 \\ 0.40740 & 0.57546 & 0.16747 \\ 0.85000 & 0.15912 & 0.58263 \\ 0.58083 & 0.86475 & 0.14074 \\ 0.16799 & 0.40081 & 0.57713 \\ 0.41156 & 0.16033 & 0.84544 \\ 0.87602 & 0.57680 & 0.40270 \\ 0.87559 & 0.40193 & 0.57506 \\ 1.67003 & -0.03139 & 0.60351 \\ 1.85152 & -0.13815 & 0.42887\end{array}$




$\begin{array}{lll}0.07905 & 0.73299 & 0.92389 \\ 0.26870 & 0.87872 & 0.74720 \\ 0.92529 & 0.26118 & 0.08004 \\ 0.73325 & 0.26180 & 0.93316 \\ 0.07178 & 0.93752 & 0.25844 \\ 0.26692 & 0.73882 & 0.08480 \\ 0.92213 & 0.08668 & 0.73209 \\ 0.72879 & 0.93197 & 0.07539 \\ 0.09790 & 0.24711 & 0.72702 \\ 0.26855 & 0.08237 & 0.90658 \\ 0.94652 & 0.72231 & 0.25318 \\ 0.93376 & 0.26166 & 0.73271 \\ 0.25906 & 0.93751 & 0.07122 \\ 0.08339 & 0.73964 & 0.26712 \\ 0.73270 & 0.08730 & 0.92052 \\ 0.72798 & 0.24740 & 0.09710 \\ 0.07643 & 0.93085 & 0.72894 \\ 0.25191 & 0.72067 & 0.94910 \\ 0.90752 & 0.08275 & 0.26796 \\ 0.08097 & 0.26128 & 0.92414 \\ 0.74517 & 0.88152 & 0.26773 \\ 0.92298 & 0.73310 & 0.07963 \\ 0.05035 & 0.05307 & 0.04982 \\ 0.05238 & 0.05061 & 0.84803 \\ 0.05163 & 0.85524 & 0.05233 \\ 0.84834 & 0.05113 & 0.05171 \\ 0.93283 & 0.93418 & 0.93279 \\ 0.91870 & 0.93804 & 0.20845 \\ 0.20841 & 0.93696 & 0.91960 \\ 0.93275 & 0.20430 & 0.93196 \\ 1.74242 & -0.16015 & 0.56773 \\ 0.12021 & 0.88041 & 0.12012 \\ 0.88154 & 0.11945 & 0.88088 \\ 0.87743 & 0.87740 & 0.12346 \\ 0.11777 & 0.11828 & 0.88270 \\ 0.88331 & 0.11860 & 0.11716\end{array}$

GB Degradation Product from C Orientation:

POSCAR file from uio-66-prim-defect-impa-hf-c.res: C: 44 F: 1 H: 36 O: 32 P: 1 ZR: 6 14.6799000000

$\begin{array}{lll}0.8088257808 & 0.2921107631 & 0.5119911970 \\ 0.0000000000 & 0.8660402570 & 0.5058074770 \\ 0.0000000000 & 0.0000000000 & 1.0000000000\end{array}$


441363216

Direct

$\begin{array}{lll}0.91958 & 0.44535 & 0.08750 \\ 0.58881 & 0.39258 & 0.00907 \\ 0.52706 & 0.90630 & 1.58942 \\ 0.65270 & 0.84306 & 1.53790 \\ 0.70306 & 0.76318 & 1.63258 \\ 0.00840 & 0.00352 & 0.29451 \\ 0.52624 & 1.05004 & 1.35749 \\ 0.94548 & 0.43158 & 0.53472 \\ 0.01250 & 0.00756 & 0.39146 \\ 0.30135 & 0.68643 & 0.00877 \\ 0.39957 & 0.58218 & 0.01177 \\ 0.07886 & 0.91425 & 0.45258 \\ 0.00807 & 0.00669 & 0.68491 \\ 0.01055 & 0.00928 & 0.58105 \\ 0.69444 & 0.00407 & 0.99523 \\ 0.08923 & 0.44192 & 0.92074 \\ 0.59311 & 0.00647 & 0.99296 \\ 0.02227 & 0.28773 & 0.68701 \\ 0.02679 & 0.38631 & 0.58273 \\ 0.45401 & 0.92090 & 0.06880 \\ 0.30561 & 0.00664 & 0.98730 \\ 0.40554 & 0.00765 & 0.98844 \\ 0.02018 & 0.68261 & 0.29492 \\ 0.02508 & 0.57798 & 0.39386 \\ 0.44735 & 0.53471 & 0.09356 \\ 0.09047 & 0.53646 & 0.91882 \\ 0.92089 & 0.53975 & 0.08577 \\ 0.94573 & 0.10350 & 0.51880 \\ 0.54764 & 0.92025 & 0.07081 \\ 0.10796 & 0.43652 & 0.53523 \\ 0.45089 & 0.09398 & 0.91062 \\ 0.10678 & 0.53253 & 0.44120 \\ 0.94493 & 0.52685 & 0.44037 \\ 0.54448 & 0.09342 & 0.91294 \\ 0.54159 & 0.43984 & 0.09234 \\ 0.07814 & 0.91538 & 0.54681 \\ 0.44539 & 0.53386 & 0.92971 \\ 0.54012 & 0.43946 & 0.92803 \\ 0.94747 & 0.10276 & 0.42375 \\ 0.00580 & 0.68845 & 0.99819 \\ 0.00604 & 0.58586 & 0.00105 \\ 0.00109 & 0.29691 & 0.00426\end{array}$




$\begin{array}{lll}0.00343 & 0.39641 & 0.00469 \\ 0.69246 & 0.29397 & 0.00535 \\ 0.62694 & 1.19206 & 1.00119 \\ 0.51868 & 0.95407 & 1.63639 \\ 0.47995 & 0.84436 & 1.65026 \\ 0.66318 & 0.79276 & 1.49228 \\ 0.79634 & 0.71778 & 1.59501 \\ 0.69196 & 0.81312 & 1.67880 \\ 0.65814 & 0.69964 & 1.69381 \\ 0.48908 & 0.96807 & 1.52025 \\ 0.48155 & 1.11077 & 1.40604 \\ 0.50677 & 1.09427 & 1.27637 \\ 0.49526 & 0.97547 & 1.41104 \\ 0.68207 & 1.12682 & 1.04947 \\ 0.26463 & 0.90475 & 0.66844 \\ 0.41657 & 0.85458 & 0.12861 \\ 0.16816 & 0.57468 & 0.40304 \\ 0.58189 & 0.15980 & 0.85340 \\ 0.58148 & 0.40094 & 0.15470 \\ 0.12849 & 0.84342 & 0.59699 \\ 0.40531 & 0.57348 & 0.86730 \\ 0.89951 & 0.17601 & 0.37186 \\ 0.15399 & 0.40146 & 0.85747 \\ 0.85636 & 0.58035 & 0.14905 \\ 0.90523 & 0.27857 & 0.90654 \\ 0.90916 & 0.90274 & 0.90310 \\ 0.29370 & 0.90604 & 0.88477 \\ 0.89150 & 0.90273 & 0.28893 \\ 0.15617 & 0.57424 & 0.85392 \\ 0.85356 & 0.40797 & 0.15225 \\ 0.57889 & 0.40061 & 0.86398 \\ 0.12952 & 0.84132 & 0.42485 \\ 0.40932 & 0.57438 & 0.15678 \\ 0.89467 & 0.17640 & 0.54667 \\ 0.58815 & 0.85312 & 0.13189 \\ 0.17029 & 0.39949 & 0.57467 \\ 0.41075 & 0.16106 & 0.84929 \\ 0.88232 & 0.56516 & 0.40086 \\ 0.88266 & 0.39083 & 0.57409 \\ 0.75290 & 0.90125 & 1.24627 \\ 0.71161 & 0.92801 & 1.45576 \\ 0.27416 & 0.88125 & 0.73905\end{array}$




$\begin{array}{lll}0.92032 & 0.25929 & 0.08045 \\ 0.73665 & 0.25765 & 0.92424 \\ 0.08231 & 0.92360 & 0.25729 \\ 0.27074 & 0.73583 & 0.07601 \\ 0.93002 & 0.08282 & 0.72688 \\ 0.73161 & 0.92560 & 0.07298 \\ 0.10110 & 0.24369 & 0.72661 \\ 0.26985 & 0.08095 & 0.90569 \\ 0.94849 & 0.71562 & 0.25264 \\ 0.93847 & 0.25530 & 0.73213 \\ 0.26262 & 0.93211 & 0.06699 \\ 0.08861 & 0.73196 & 0.26196 \\ 0.73728 & 0.08039 & 0.91773 \\ 0.73112 & 0.25390 & 0.08327 \\ 0.08371 & 0.92719 & 0.72451 \\ 0.25697 & 0.71980 & 0.93852 \\ 0.92881 & 0.07921 & 0.25838 \\ 0.07994 & 0.25694 & 0.92592 \\ 0.92696 & 0.72861 & 0.07604 \\ 0.04966 & 0.04706 & 0.05069 \\ 0.05588 & 0.04615 & 0.84615 \\ 0.05588 & 0.84845 & 0.04829 \\ 0.84813 & 0.04529 & 0.05254 \\ 0.93673 & 0.92926 & 0.92974 \\ 0.92491 & 0.92892 & 0.20682 \\ 0.21168 & 0.93472 & 0.91533 \\ 0.93400 & 0.19749 & 0.93310 \\ 0.68173 & 0.99957 & 1.32383 \\ 0.12754 & 0.87357 & 0.87409 \\ 0.12249 & 0.87587 & 0.11665 \\ 0.88497 & 0.11387 & 0.88004 \\ 0.88230 & 0.87135 & 0.12036 \\ 0.11806 & 0.11410 & 0.88448 \\ 0.87756 & 0.11363 & 0.12206\end{array}$

INCAR File for Optimizations:

ISTART $=0$

$I C H A R G=0$

$I S I F=2$

ENCUT $=400$ 


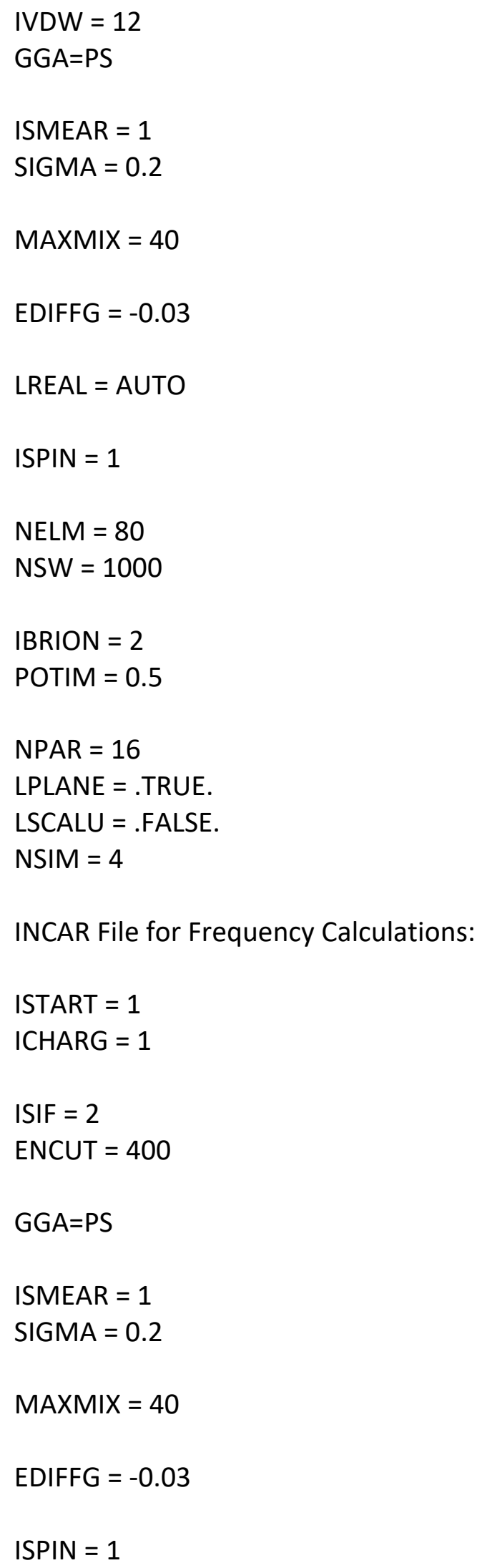




$$
\begin{aligned}
& \text { ISYM }=0 \\
& \text { NELMIN }=10 \\
& \text { NELM }=120 \\
& \text { NSW }=1 \\
& \text { LREAL }=. \text { FALSE. } \\
& \text { NWRITE }=3 \\
& \text { IBRION }=7 \\
& \text { LEPSILON =.TRUE. } \\
& \text { POTIM }=0.02 \\
& \text { LPLANE = .TRUE. } \\
& \text { LSCALU = .FALSE. } \\
& \text { NSIM }=4 \\
& \text { NPAR }=16
\end{aligned}
$$

